\title{
Corela
}

Cognition, représentation, langage

HS-20 | 2016

L'Implicite

\section{Négociation interactive de l'implicite dans le débat polémique radiophonique}

\section{Bernadeta Wojciechowska et Elisabeth Richard}

\section{OpenEdition}

\section{Journals}

Édition électronique

URL : http://journals.openedition.org/corela/4684

DOI : $10.4000 /$ corela.4684

ISSN : $1638-573 \mathrm{X}$

Éditeur

Cercle linguistique du Centre et de I'Ouest - CerLICO

Référence électronique

Bernadeta Wojciechowska et Elisabeth Richard, « Négociation interactive de l'implicite dans le débat polémique radiophonique », Corela [En ligne], HS-20 | 2016, mis en ligne le 06 décembre 2016, consulté le 01 mai 2019. URL : http://journals.openedition.org/corela/4684; DOI : 10.4000/corela.4684

Ce document a été généré automatiquement le 1 mai 2019.

\section{(c) (1) (3) (-)}

Corela - cognition, représentation, langage est mis à disposition selon les termes de la licence Creative Commons Attribution - Pas d'Utilisation Commerciale - Partage dans les Mêmes Conditions 4.0 International. 


\title{
Négociation interactive de l'implicite dans le débat polémique radiophonique
}

\author{
Bernadeta Wojciechowska et Elisabeth Richard
}

1 http://www.franceinter.fr/emission-service-public-nos-politiques-ont-ils-dejarencontre-le-peuple-0

\section{Introduction}

2 La réflexion que nous proposons se situe dans le prolongement des travaux que nous menons depuis maintenant quelques années conjointement entre les enseignants et apprenants de Français Langue Étrangère (désormais FLE) de l'université de Poznań en Pologne $e^{1}$ et les enseignants et étudiants des masters FLE, futurs formateurs de FLE, à Rennes 2, en France ${ }^{2}$. C'est donc dans une perspective linguistique et didactique que nous plaçons notre objet d'étude et, plus précisément, nous interrogeons ici la notion d'implicite et ses incidences pour une didactique rénovée de la compétence de compréhension orale pour les niveaux avancés et très avancés $\left(\mathrm{C} 1 / \mathrm{C} 2 \mathrm{du} \mathrm{CECRL}^{3}\right)$.

\section{Didactique de la compréhension orale}

\section{I.1 Dépasser l'écoute de restitution et les implicites encyclopédiques}

3 Nos recherches précédentes sur la didactique de la compréhension du débat radiophonique montrent la difficulté pour les étudiants de niveaux très avancés $\left(\mathrm{C} 1 / \mathrm{C2}^{4}\right)$ de dépasser une démarche de restitution, omniprésente dans les tâches d'écoute jusqu'aux niveaux $\mathrm{B} 1 / \mathrm{B}^{5}$. Ces tâches d'écoute, qui privilégient le repérage et l'accumulation des informations dans une approche par thèmes, ne prennent, à notre sens, pas suffisamment en compte le caractère non linéaire, dynamique et co-construit du 
discours à l'oral. En outre, l'analyse des consignes d'activités d'écoute met en évidence l'insuffisance des propositions didactiques concernant l'oral radiophonique, propositions qui restent le plus souvent calquées sur les textes écrits (type articles de presse). Ces activités imposent une démarche de compréhension basée sur les aspects linguistiques, textuels et référentiels sans les intégrer dans une construction interactive plus large et sans tenir suffisamment compte de la dimension sociale et finalisée (intentionnelle) du discours 6 .

4 C'est ainsi qu'on peut expliquer en partie la très faible prise en compte des implicites, pourtant nécessaires à l'interprétation du discours de débat radiophonique. En effet, dans la didactique du FLE, la notion d'implicite est majoritairement associée aux implicites culturels et encyclopédiques: les noms propres par exemple jouent un rôle parfois déstabilisant en ce sens dans la compréhension immédiate. Pourtant, il nous semble que ces implicites culturels, de type Npropres, sont relativement faciles non seulement à repérer au niveau $\mathrm{C} 1 / \mathrm{C} 2$, mais encore à combler, entre autres par la possibilité de recherche immédiate offerte aujourd'hui par Internet; dans la plupart des cas, ils ne relèvent pas exclusivement d'une défaillance de compréhension à l'oral puisqu'à l'écrit aussi les apprenants seront confrontés à ces lacunes de type encyclopédique. ${ }^{7}$

\section{I.2 Construire des compétences interprétatives}

5 Ce qui s'avère plus difficile à saisir pour l'apprenant dans la linéarité du flux sonore, ce sont les implicites que l'on appellera ici implicites discursifs et implicites sociodiscursifs.

6 On entendra par implicite(s) discursif(s), les représentations partagées relatives aux types de discours (ici discours médiatique) et aux genres (débat, interview pour ne prendre que ces deux genres). Les dispositifs sous-jacents sont loin d'être familiers pour les étudiants étrangers qui n'ont pas la même culture du débat, et encore moins la même expérience du débat et du débat radiophonique en particulier.

7 Quant aux implicites socio-discursifs, on les envisagera dans le cadre restreint de cet article sous deux angles: d'un côté le choix, le rôle institutionnel des invités et leur négociation tout au long de l'émission; de l'autre, la formulation initiale de la problématique et son traitement interactif.

8 L'objectif de notre travail est donc d'envisager un enseignement de la compréhension orale basé sur une analyse distanciée qui prenne en compte les normes implicites des genres de discours et leur réalisation concrètes dans le discours. Cela revient à dire qu'on étudiera ici les endroits du discours où ces normes implicites sont soit explicitées soit négociées par les différents intervenants du débat.

9 Pour ce faire ${ }^{8}$, nous avons choisi de travailler une émission quotidienne de France Inter, "Service public ", émission qui se veut être un débat radiophonique sur des questions d'actualité sociale. On prendra en particulier appui sur l'émission du 24 octobre 2014 intitulée « Nos politiques ont-ils rencontré le peuple? »".

Dans l'analyse de l'émission, on s'intéressera prioritairement aux stratégies de négociation des implicites discursifs et socio-discursifs, pour ainsi dire à la croisée de l'intentionnalité et de la convention discursive, en les considérant comme des endroits sur lesquels l'attention des apprenants devrait être focalisée pour construire des compétences interprétatives. 


\section{L'implicite discursif : le genre « débat radiophonique "}

\section{II.1. Normes implicites et négociation des genres discursifs}

11 Si l'on définit le débat comme « une confrontation d'opinions à propos d'un objet particulier (...) qui se déroule dans un cadre pré-fixé » (Kerbrat Orecchioni (1990 : 118)), notre émission radiophonique relève bien de ce genre discursif : un journaliste ${ }^{10}$ gère conjointement l'espace, le temps, le temps et la distribution de la parole, et, a priori tout au moins, la progression thématique.

12 L'émission "Service public » est une émission récurrente avec ses habitudes, ses règles organisationnelles propres, sa longueur prédéterminée (environ 50 minutes d'antenne), ses coupures musicales (ou autres) et nous avons déjà montré (Richard et al. 2013) qu'une fréquentation régulière permet à l'apprenant de repérer et de s'approprier cette organisation pour (mieux) s'en dégager et se consacrer pleinement à ce qui fait débat. Pour autant, c'est bien l'« hybridation » des genres (au sens de Cosnier et Kerbrat 1987) qui est à l'œuvre tout au long du discours, alternant ainsi des séquences de débat polémique (au final assez peu dans notre émission), des séquences qui relèveraient de l'interview et des séquences qui relèveraient plutôt de la discussion (non polémiques).

13 Ces jeux de codes alternatifs de schémas discursifs sont des phénomènes bien connus des linguistes qui peuvent décrire comment sur du long terme se déplient et s'imbriquent ces différents schémas. Mais c'est un apprentissage absent des manuels et la reconnaissance de ces différentes séquences ne fait pas partie des tâches de compréhension orale. Pourtant la mise au jour de cette hybridation singulière, constante, on peut même dire constitutive de la langue à l'oral permettrait aux apprenants d'être plus à l'aise dans la reconnaissance des normes implicites à chaque genre et ainsi de mieux saisir les enjeux énonciatifs et argumentatifs qui s'y déploient.

\section{II.2 Des moments d'écoute pertinents}

14 On peut ainsi montrer que la prise en compte de certains «endroits/moments » du discours et de certaines formes particulières permettrait à l'apprenant de repérer des schémas discursifs distincts et d'en tirer des conséquences interprétatives singulières. C'est notamment le cas, en tout cas on en a des indices forts, dans les modes de prises de parole des uns et des autres. Ainsi, une attention particulière portée aux formes d'introduction de la parole permet d'intégrer la séquence dans une démarche polémique ou au contraire l'inscrit dans une continuité énonciative.

\section{II.2.1 Le genre « Interview »}

Dans notre émission, rares sont les prises de parole polémiques. Dans la majorité des cas, le journaliste s'adresse à un invité sur le mode de l'interview ${ }^{11}$, et l'invité s'inscrit à son tour dans ce genre discursif :

Journaliste : \#1 mais si l'on vous suit et si l'on considère que ces diners en ville sont les lieux où finalement se rassemblent les gens qui comptent en ville pour accéder à

la table à la table du diner il faut avant tout avoir des diplômes appartenir à une 
noblesse d'État comme le disait Bourdieu autrement dit e ne dinent là-bas que des énarques des hauts fonctionnaires des personnes qui ont déjà été sélectionnées par le système et dans ces conditions vous dépeignez finalement un monde complètement fermé qui qui n'a e qu'une ambition celle de conserver le pouvoir quel que soit le pouvoir \#

Raphaëlle Baqué : \#2 si vous voulez cette endogamie n'est pas nouvelle hein e mais ce qui frappe c'est l'importance qu'elle a prise et et je pense que ce que disait Michèle Delaunay à vrai dire c'était ça c'est c'est la modification aujourd'hui effectivement e on on trouve en politique plus qu'avant e non seulement e ceux qui appartiennent aux grands corps les énarques et Dieu sait qu'on on sait bien cette critique e est constante depuis déjà cinquante ans à vrai dire mais aussi les apparatchiks du parti par exemple qui ont une importance tout à fait considérable et \#

Journaliste : \#1 plus qu'avant? \#

Raphaëlle Baqué : \#2 plus qu'avant oui bien sûr \#

Journaliste : \#1 pourquoi \#

Raphaëlle Baqué : \#2 parce que le parti est devenu un système de sélection et qu'effectivement on voit aujourd'hui des gens qui arrivent dans les cabinets où même e à à l'Assemblée Nationale on on on voit bien ces ces nouveaux élus qui n'ont effectivement comme le disait Michèle Delaunay tout à l'heure pas d'expérience du réel c'est pas tellement une question de classe sociale si vous voulez encore que évidemment ça se recoupe mais ce n'est seulement ça ce n'est pas seulement ça c'est-à-dire que ce sont des gens qui ont qui ont fait toute leur carrière e comme assistant parlementaire ou e au sein du parti et qui n'ont par exemple jamais travaillé en entreprise et ça effectivement on le voit ça ça l'homogénéité si vous voulez s'est accentuée \#

Il y a en somme comme une continuité discursive et énonciative qui s'instaure entre le journaliste et son invité. Le journaliste formule une suite de questions dont la fonction essentielle est de permettre à l'invitée de présenter et d'expliquer son point de vue (arguments ou faits) aux auditeurs. On est dans une co-construction discursive collaborative : la construction syntaxique en est un indice fort puisque l'invitée s'inscrit dans la syntaxe du journaliste soit par répétition à l'identique «-Plus qu'avant? -plus qu'avant oui bien sûr", soit par subordination "-Pourquoi ? - parce que le parti est devenu un système de sélection et que... ».

\section{II.2.2 De l'interview au débat}

17 Néanmoins, parfois, l'invité n'adhère pas au genre requis. Il s'en démarque. C'est ce moment du discours qu'il faut être capable de repérer. Dans notre émission, la première invitée à qui le journaliste donne la parole est Michèle Delaunay. Le journaliste vient de formuler sa propre définition du débat du jour «Les politiques ont-ils rencontré leur peuple? » dans le chapeau de l'émission. La présentation qu'il fait de Michèle Delaunay et du billet qu'elle vient du publier sur son blog semblent aller dans le sens du témoignage de cette vision de la problématique. Pourtant, dès sa première prise de parole, Michèle Delaunay s'inscrit en faux par rapport à cette vision de la thématique. On peut dire qu'elle discute (qu'elle dispute) le fondement même de la thématique telle qu'elle a été proposée par le journaliste :

Michèle Delaunay : bonjour \#

Journaliste : vous êtes députée PS de la Gironde conseillère municipale de Bordeaux ancienne ministre déléguée chargée des personnes âgées et de l'étonn et de l'autonomie et vous avez publié sur votre Blog un billet intitulé Le Tunnel ou comment faire carrière sans mettre un pied dans la vraie vie où vous expliquez que 
le milieu politique est un milieu extrêmement fermé qui vit dans l'entre-soi selon vous de quoi se compose ce tunnel Michèle Delaunay \# Michèle Delaunay: \#4 alors vous posez une question un tout petit peu différente de ce qui était la mienne moi je je disais la classe politique n'a pas rencontré suffisamment le réel le peuple est une notion e qui a deux sens la classe populaire les plus pauvres et deuxièmement le peuple français dans toute sa diversité les deux questions ont un sens

Pour l'apprenant, il s'agit de repérer ce basculement des genres discursifs pour comprendre et interpréter que Michèle Delaunay inscrit sa parole dans un autre débat que celui auquel l'animateur l'enjoint. Il s'agit là d'un point de repère indispensable à l'interprétation des différents échanges qui auront lieu entre le journaliste et cette intervenante tout au long de l'émission. Ainsi, par exemple, sept tours de parole plus tard, le journaliste cherche à nouveau à ramener Michèle Delaunay dans son propre débat:

Journaliste : \#1 Michèle Delaunay vous n'avez pas répondu à ma question en quoi le gouvernement qui n'appartenait pas donc e qui n'était pas issu de la classe populaire ou qui n'en venait pas en quoi celui-ci était coupé de la réalité sociale du pays \#

Cette fois, $\mathrm{M}$. Delaunay répond sur le mode de la polémique en renversant l'argument du journaliste par un «si j'ai répondu, pardonnez-moi, car... » et c'est le marqueur de justification CAR qui affirme et confirme la motivation des développements énonciatifs auxquels elle s'est prêtée jusque-là :

Michèle Delaunay : \#4 alors je je si j’ai répondu pardonnez-moi car je crois que le gouvernement ne pas être...

Tout l'enjeu de la compréhension orale se situe dans la capacité à suivre ces va-et-vient discursifs et argumentatifs au sein du même débat radiophonique. Identifier au sein des interventions ce qui relève et/ou dépasse le cadre discursif préfixé (ici l'interview) doit permettre à l'apprenant d'orienter son analyse sur les points de divergence entre les deux interlocuteurs plutôt que, comme c'est souvent le cas, d'adopter simplement l'évaluation du journaliste « vous n'avez pas répondu à ma question ». Suivre les modes de réponse de Michèle Delaunay, c'est saisir les enjeux du débat (comprendre qu'il y a débat) et comprendre dans le même temps la posture discursive - coopérative ou non - du journaliste.

\section{II.3 À l'écoute des connecteurs}

21 Le jeu des connecteurs argumentatifs et autres marqueurs discursifs ${ }^{12}$ est également intéressant à observer.

22 Au CAR qui justifie en imposant une vérité, et qui n'est utilisé que par Michèle Delaunay dans ce débat ( 5 occ.), viendront bientôt se confronter les « effectivement, et si vous voulez " de la journaliste Raphaëlle Baqué, deuxième invitée de l'émission à prendre la parole ${ }^{13}$. 
Journaliste: [...] Raphaëlle Bacqué grand reporter au Monde vous avez publié avec Ariane Chemin Raphaëlle Baqué un portrait des Époux Jouyet les Époux Jouyet c'est tout d'abord pour monsieur Jouyet qui est secrétaire général de l'Élysée et également pour sa femme l'une des dirigeante de Sciences Po et vous dites ils tutoient le pouvoir de droite et de gauche ils incarnent cette bourgeoisie d'État promue par leur ami Hollande et ce portrait est de mon point de vue un portrait au vitriol d'une classe d'État complètement coupée du peuple vous ai-je bien lu \#

Raphaëlle Bacqué: oui enfin ce qui nous a frappées effectivement à à vrai dire on a un peu découvert ça e en en enquêtant c'était effectivement l'am l'ampleur d'un d'un réseau hein e amical e e aussi e corporatiste si vous voulez parce que e Jean-Pierre Jouyet est issu $\mathrm{du} d u$ du corps de l'inspection des finances e Brigitte e Taittinger Jouyet elle est issue d'une grande famille hein e la famille Taittinger qui est une famille qui a possédé e un très grand groupe e e notamment e de luxe et et les champagnes aujourd'hui e et effectivement les les le l'am la la la réunion de leur deux réseaux e occupent au fond tout une grande partie des c'est ils font une grande partie des nominations d'aujourd'hui de l'État et c'est vrai qu'il est sem parfois plus utile et plus efficace de diner à leur table que de faire e e e de la politique ou de diriger e de d'avoir une action publique e différente \#

Journaliste: mais si l'on vous suit et si l'on considère que ces diners en ville sont les lieux où finalement se rassemblent les gens qui comptent en ville pour accéder à la table à la table du diner il faut avant tout avoir des diplômes appartenir à une noblesse d'État comme le disait Bourdieu autrement dit e ne dinent là-bas que des énarques sélectionnées par le système et dans ces conditions vous dépeignez finalement un monde complètement fermé qui qui n'a e qu'une ambition celle de conserver le pouvoir quel que soit le pouvoir \#

Raphaëlle Bacqué: si vous voulez cette endogamie n'est pas nouvelle hein e mais ce qui frappe c'est l'importance qu'elle a prise et et je pense que ce que disait Michèle Delaunay à vrai dire c'était ça c'est c'est la modification aujourd'hui effectivement e on on trouve en politique plus qu'avant e non seulement e ceux qui appartiennent aux grands corps les énarques et Dieu sait qu'on on sait bien cette critique e est constante depuis déjà cinquante ans à vrai dire mais aussi les apparatchiks du parti par exemple qui ont une importance tout à fait considérable et \#

Journaliste: plus qu'avant? \# / ,

Raphaëlle Bacqué: plus qu'avant oui bien sûr \# Journaliste: pourquoi \#

Raphaëlle Bacqué: parce que le parti est devenu un système de sélection et qu' effectivement on voit aujourd'hui des gens qui arrivent dans les cabinets où même e à à l'Assemblée Nationale on on voit bien ces ces nouveaux élus qui n'ont effectivement comme le disait Michèle Delaunay tout à l'heure pas d'expérience du réel c'est pas tellement une question de classe sociale $\underline{\mathbf{s i}}$ vous voulez encore que évidemment ça se recoupe mais ce n'est seulement ça ce n'est pas seulement ça c'est-à-dire que ce sont des gens qui ont qui ont fait toute leur carrière e comme assistant parlementaire ou e au sein du parti et qui n'ont par exemple jamais travaillé en entreprise et ça effectivement on le voit ça ça l'homogénéité si vous voulez s'est accentuée \#

23 Ces marqueurs sont omniprésents dans le discours de Raphaëlle Bacqué, on peut même dire qu'ils sont sur-représentés si on les compare aux autres marqueurs présents dans son discours :

Connecteurs Raphaëlle Bacqué 


\begin{tabular}{|l|l|l|l|l|}
\hline Effectivement & Si vous voulez & Car & Parce que & Puisque \\
\hline 14 & 10 & 0 & 3 & 0 \\
\hline
\end{tabular}
discursifs qui se jouent dans cette émission, et on peut dire que Raphaëlle Bacqué joue le jeu du « débat » dans le sens où non seulement elle exhibe avec effectivement l'histoire discursive en cours, mais on peut dire encore qu'avec si vous voulez, elle inscrit son discours explicatif dans une collaboration avec l'auditeur. En somme la co-construction du discours dans et par l'émission elle-même se double d'une prise en compte et d'une exploitation de l'Autre, on pourrait dire de tous les Autres (journaliste, invités, mais aussi auditoire et auditeurs), tous acteurs du discours en train de se tenir.

\section{L'implicite socio-discursif : le jeu des rôles}

\section{III.1 Des images préalables et de leur activation dans le discours}

29 On sait depuis Aristote ${ }^{15}$ que l'évaluation de la pertinence des propos du locuteur et l'adhésion du public à son argumentation (sa force illocutoire) sont tributaires de l'image projetée et/ou construite par le locuteur. Celle-ci se compose grosso modo des rôles institutionnels revendiqués et de l'image personnelle (caractère, culture, ethnie, etc.) du locuteur.

Dans le débat radiophonique, les rôles sociaux-discursifs interviennent à trois niveaux différents mais indubitablement liés et dynamiques : 1) les rôles préalables au discours; 2) les rôles actualisés dans le discours par le journaliste dans son introduction en fonction de la scène générique et du choix d'une scénographie; 3) les rôles négociés et/ou 
revendiqués par les intervenants eux-mêmes. Au niveau discursif l'image découle d'abord de la distribution des rôles ${ }^{16}$.

31 Avant même toute prise de parole, l'image du locuteur est d'abord construite par le journaliste. Cette identité s'appuie sur ce que R. Amossy ${ }^{17}$ désigne comme ethos préalable: c'est l'image qui découle d'une part des rôles sociaux, des fonctions et du statut assumés par le locuteur dans l'espace social et d'autre part, des représentations collectives, des valeurs et de la légitimité conférées à ceux-ci dans une société donnée - on pourrait ajouter, pour des personnalités reconnues du grand public, des représentations de l'identité personnelle.

Côté apprentissage, autant mettre en rapport les énoncés des locuteurs avec leur positionnement argumentatif dans le discours polémique semble accessible au niveau B2/ C1, autant faire un lien entre les énoncés et les rôles institutionnels actualisés, et évaluer la légitimité du locuteur apparaît comme une tâche beaucoup plus exigeante.

Deux contraintes implicites sont à prendre en compte : d'une part, comme le rappelle $\mathrm{R}$. Amossy, l'image du locuteur " est en prise sur le modèle culturel de l'époque ${ }^{18}$ "; d'autre part, il faut encore considérer que les différentes facettes du locuteur seront activées/ transformées/rejetées à différents moments du discours.

aspect nous semble plus exigeant encore : il s'agit pour l'auditeur de saisir comment les locuteurs se servent de leurs rôles pour légitimer leur parole (ou, parfois, celle des autres).

Pour mieux cerner les enjeux propres à ces différents pans d'analyse des implicites sociodiscursifs dans notre débat radiophonique, nous nous concentrerons ici sur le choix des invités et leur présentation initiale, et nous observerons comment se négocient, dans l'interaction, les différents rôles.

\section{III.2. Le choix et la présentation des invités : indice de stratégie argumentative}

Observer la présentation des invités, c'est aussi prendre conscience de la justification implicite de leur présence dans ce débat. Tous les invités sont dits avoir un lien avec la thématique retenue ce jour, mais sur un mode qui les distingue néanmoins.

La première ${ }^{19}$ invitée présentée par le journaliste est Raphaëlle Bacqué :

Journaliste : \#1 Raphaëlle Baqué vous êtes e grand reporter au quotidien Le Monde vous avez notamment publié avec Ariane Chemin un grand portrait des époux Jouyet les époux Jouyet étant donc le secrétaire de l'Élysée pour Jean-Pierre Jouyet et sa femme qui est directrice de la communication de Sciences Po et vous racontez finalement un certain milieu parisien un certain milieu politique en face de vous se trouve Jacques Julliard bonjour Jacques Julliard \#

Les qualités professionnelles de l'invitée sont posées d'entrée de jeu, elle est «grand reporter" dans un grand quotidien national français Le Monde, deux gages qui garantissent l'autorité (objective?) de sa parole. Une fois l'autorité énonciative posée, vient un second argument qui justifie sa présence immédiate, dans ce débat en particulier : la publication récente d'un " portrait des époux Jouyet ».

Après une explication sur le mode objectif des dits époux Jouyet, le journaliste se fait commentateur de sa propre lecture du portrait «vous racontez finalement un certain milieu parisien, un certain milieu politique». La recatégorisation sur le mode évaluatif 
doit susciter la curiosité des auditeurs, générer des attentes sur les résultats de l'enquête, mais aussi garantir en quelque sorte la vraisemblance des révélations rapportées.

Le deuxième invité à être présenté est Jacques Julliard :

Journaliste: \#1 vous avez beaucoup publié vous êtes un penseur du politique vous votre dernier ouvrage s'intitule les gauches françaises figures et paroles aux Éditions Flammarion et vous avez également publié avec Jean-Claude Michéa la gauche et le peuple aux Éditions Flammarion et puis en duplex de Bordeaux Michèle Delaunay bonjour Michèle Delaunay \#

41 Dans cette présentation, Guillaume Erner fait abstraction de la profession de journaliste qui a été celle de Jacques Julliard, de son passé de militant syndicaliste, d'enseignant d'histoire. Cette présentation atemporelle (contrairement aux deux autres) fait de cet invité un observateur, un analyste de la vie politique: «vous êtes un penseur du politique ». Présenter Jacques Julliard comme un penseur c'est lui donner un statut plus élevé que celui de reporter, surtout si ce statut est confirmé par les publications de l'invité, ce dont on a déjà été immédiatement avertis : « Vous avez beaucoup publié ».

Les attentes que ce rôle de penseur peut générer chez les auditeurs sont celles de l'éclairage, de l'analyse (en termes de causes et conséquences pour la vie sociale et politique) des informations rapportées par les autres invités. Donner le statut d'un penseur au locuteur, c'est aussi lui donner un statut neutre et non engagé, ce qui s'avérera par la suite non seulement modifié mais démenti, en partie dans et par les propos de Jacques Julliard lui-même.

Sans entrer dans les détails, disons seulement que le discours de Julliard porte de nombreuses traces de son engagement dans l'action politique et dépasse largement le rôle de penseur et d'observateur de la vie politique. Ces traces se concrétisent dans la matérialité linguistique : Jacques Julliard devient par moment franchement militant et revendicatif "je demande aux politiques d'ouvrir la profession politique", "il faut empêcher la représentation politique de se constituer en classe », «il faut une nuit de 4 août de la classe politique ».

Dans la perspective de l'interprétation de l'implicite par les étudiants, il est intéressant de remarquer que cette posture de revendication est explicitement pointée dans un commentaire de Raphaelle Baqué :

Jacques Julliard : \#2 et e oui on a connu la Commune de Paris ça a conn ça a duré 40

jours

Raphaëlle Baqué : \#2 Jacques est pour la Révolution (rires)

La troisième et la dernière invitée à être présentée est Michèle Delaunay. Le journaliste lui attribue le statut de politicienne ayant une riche expérience de la vie politique à plusieurs niveaux, listés dans leur ordre chronologique, du plus actuel au plus ancien, "vous êtes députée PS de la Gironde conseillère municipale de Bordeaux ancienne ministre...». Ce qui justifie sa présence aujourd'hui, c'est à nouveau une actualité d'écriture, un billet sur son blog. Et c'est ce regard critique porté de l'intérieur sur l'univers politique qui engagera le début du débat qui semble d'entrée de jeu confirmer l'orientation du titre et de l'introduction de le journaliste.

Journaliste: \#1 vous êtes députée PS de la Gironde conseillère municipale de Bordeaux ancienne ministre déléguée chargée des personnes âgées et de l'étonn et de l'autonomie et vous avez publié sur votre Blog un billet intitulé Le Tunnel où comment faire carrière sans mettre un pied dans la vraie vie où vous expliquez que le milieu politique est un milieu extrêmement fermé qui vit dans l'entre-soi selon vous de quoi se compose ce tunnel Michèle Delaunay \# 
Pour résumer, on peut dire que la scénographie est construite autour de trois invités assumant des rôles complémentaires: une invitée qui porte un regard d'enquêteur au service du citoyen, extérieur au milieu politique mais qui l'a côtoyé de près pour mener son enquête (c'est Raphaëlle Bacqué), une invitée qui connaît la problématique de l'intérieur, qui en a fait l'expérience en tant que politicienne (Michèle Delaunay) et un invité plus distancié, capable de porter un regard analytique (à savoir, Jacques Julliard). Tous légitimés, autorisés à parler et à prendre part au débat.

\section{III.3 L'intégration du discursif et socio-discursif au service d'une stratégie argumentative}

Les choix de cadrage doivent être envisagés par les apprenants comme des indices de l'orientation argumentative impulsée, en tout cas, proposée par le journaliste.

Pour autant, ce cadre préconstruit ne reste pas immuable tout au long du débat: il est soumis à de constantes négociations. L'accès à la compréhension de ces négociations constituera une aide précieuse pour l'interprétation du discours et la prise de conscience de caractère préfixé et orienté du débat radiophonique. L'observation des négociations peut fournir aussi des outils intellectuels et discursifs d'évaluation de la pertinence de la scénographie mise en œuvre par les locuteurs experts ${ }^{20}$, donc modèles pour l'apprenant de langue étrangère. Pour illustrer ce pan de l'analyse, on peut revenir au premier échange entre le journaliste et Michèle Delaunay, séquence où l'on retrouve de nombreuses tentatives de dépassement du cadre instauré :

Journaliste : vous êtes députée PS de la Gironde conseillère municipale de Bordeaux ancienne ministre déléguée chargée des personnes âgées et de l'étonn et de l'autonomie et vous avez publié sur votre Blog un billet intitulé Le Tunnel ou comment faire carrière sans mettre un pied dans la vraie vie où vous expliquez que le milieu politique est un milieu extrêmement fermé qui vit dans l'entre-soi selon vous de quoi se compose ce tunnel Michèle Delaunay \#

Michèle Delaunay : alors vous posez une question un tout petit peu différente de ce qui était la mienne moi je je disais la classe politique n'a pas rencontré suffisamment le réel le peuple est une notion e qui a deux sens la classe populaire les plus pauvres et deuxièmement le peuple français dans toute sa diversité les deux questions ont un sens il est vrai qu'il n'y a pas suffisamment de diversité sociale dans la classe politique si j'ose dire la représentant d'un certain point de vue puisque je ne suis pas de classe populaire mais ce que j'exprime c'est que il faut aussi rencontrer la réalité de la vie et là je dois dire que je considère comme pratiquement toutes les femmes qui sont venues à la politique à cause de la loi sur la parité ce qui est mon cas elles avaient une vie avant la politique la mienne fut quarante-cinq ans de médecine hospitalière en $\mathrm{CHU}$ et là je peux assurer que j'ai rencontré la réalité dans toute e sa gravité \#

Journaliste : justement Michèle Delaunay en quoi votre expérience donc vous étiez médecin vous l'êtes toujours d'ailleurs en quoi le fait d'être e médecin vous distingue des autres hommes et femmes politiques qui toute leur vie ont été des politiques qu'est-ce que ça provoque sur votre manière de regarder la réalité sociale du pays \#

49 À la question du journaliste, Michèle Delaunay réagit sur le mode de la discussion, et cela au moins à deux niveaux qui restent toutefois emboîtés. D'abord, elle négocie le cadre thématique proposé par le journaliste en le reprenant poliment «alors vous posez une question un tout petit peu différente de ce qui était la mienne » (cf. partie I.1) et précise son propre objet du débat: «moi je je disais la classe politique n'a pas rencontré 
suffisamment le réel ». Son argumentation à elle portera sur la proximité avec le réel. Elle introduit ainsi une nouvelle opposition, forte, entre «le réel» et "le peuple», et démontre, définitions à l'appui, que les notions ont des sens différents : «Le peuple est une notion qui a deux sens la classe populaire les plus pauvres et deuxièmement le peuple français dans toute sa diversité ». Elle précise également que son billet sur son blog concernait la rencontre du réel à travers une activité professionnelle quelle qu'elle soit et non l'origine sociale. Elle légitime son approche en se référant à sa propre identité sociale qui n'est pas dit «du peuple» "puisque je ne suis pas de classe populaire». Elle revendique également son rôle de femme avant d'être une femme politique ${ }^{21}$ "elles avaient une vie avant la politique». Mais son argument le plus fort, son topic, elle le donne en dernier : c'est son expérience professionnelle « quarante-cinq ans de médecine hospitalière en $\mathrm{CHU}$ et là je peux assurer que j'ai rencontré la réalité dans toute e sa gravité ».

50 Le journaliste se saisit alors de la part "professionnelle » de l'identité revendiquée par Michèle Delaunay elle-même pour rebondir et, avec le marqueur d'accroche justement, tente de recadrer le discours comme il l'entend et de distinguer Delaunay des autres politiques $^{22}$.

51 Notons encore que le journaliste ne retient pas les arguments contradictoires (au sens rhétorique) mis au jour par Delaunay pour renégocier la problématique en opposant le réel vs le peuple. Il n'entre pas dans ce débat, et ne le soumettra pas non plus aux autres invités.

52 C'est pourtant bien cette opposition réel/peuple (absente de la problématique du journaliste) qui permettra à Raphaëlle Bacqué de "faire débat », comme on l'a déjà dit dans la première partie, et qui trouvera là, elle aussi, matière à négocier et à développer une argumentation qui lui sera propre :

Raphaëlle Bacqué : si vous voulez cette endogamie n'est pas nouvelle hein e mais ce qui frappe c'est l'importance qu'elle a prise et et je pense que ce que disait Michèle Delaunay à vrai dire c'était ça c'est c'est la modification aujourd'hui effectivement $e$ on on trouve en politique plus qu'avant e non seulement e ceux qui appartiennent aux grands corps les énarques et Dieu sait qu'on on sait bien cette critique e est constante depuis déjà cinquante ans à vrai dire mais aussi les apparatchiks du parti par exemple qui ont une importance tout à fait considérable et \#

Journaliste : plus qu'avant? \# /,

Raphaëlle Bacqué : plus qu'avant oui bien sûr \#

Journaliste : pourquoi \#

Raphaëlle Bacqué: parce que le parti est devenu un système de sélection et qu'effectivement on voit aujourd'hui des gens qui arrivent dans les cabinets où même e à à l'Assemblée Nationale on on on voit bien ces ces nouveaux élus qui n'ont effectivement comme le disait Michèle Delaunay tout à l'heure pas d'expérience du réel c'est pas tellement une question de classe sociale si vous voulez encore que évidemment ça se recoupe mais ce n'est seulement ça ce n'est pas seulement ça c'est-à-dire que ce sont des gens qui ont qui ont fait toute leur carrière e comme assistant parlementaire ou e au sein du parti et qui n'ont par exemple jamais travaillé en entreprise et ça effectivement on le voit ça ça l'homogénéité si vous voulez s'est accentuée \# opposition qui devient dès lors première ${ }^{23}$ :

Jacques Julliard: (...) c'est vrai e que e la l'absence d'expérience professionnelle est encore plus grave que l'absence de diversité sociale mais je voudrais quand 
même souligner cette chose c'est vous voyez comme les mentalités ont changé il y a une trentaine d'années on se scandalisait enfin dans des milieux très restreints on se scandalisait e qu'il y ait si peu de représentants des des ouvriers des artisans e des employés e au Palais Bourbon à l'Assemblée Nationale e aujourd'hui personne ne s'intéresse plus à cela la la la le le seul critère c'est la parité homme femme je me réjouis qu'on aille dans le sens de la parité homme femme mais personne ne ne demande une parité entre la classe ouvrière par exemple ou ce qu'il en reste ou les classes populaires de manière générale et puis e la la la bourgeoisie e la bourgeoisie d'État \#

La problématique initiale a donc été négociée dès la première intervention, et au final l'opposition première proposée par le journaliste politique vs peuple, est devenue réel vs peuple, puis réel =professionnel vs politiques =a-professionnels. En somme, débat il y a bien eu lieu et ce débat s'est construit dans et par les différents échanges non seulement entre le journaliste et les invités mais aussi entre les invités eux-mêmes. La thématique a évolué en même temps que les rôles discursifs et socio-discursifs des invités. Il s'agit pour l'apprenant de repérer à quel point le traitement de la problématique est inséparable de la négociation des rôles qui légitiment les prises de positions.

\section{Conclusions}

L'analyse que nous avons proposée montre la complexité du débat radiodiffusé qui requiert la capacité à suivre et à intégrer les implicites discursifs et les implicites sociodiscursifs. Elle permet de pointer l'insuffisance de la didactique de la compréhension orale qui est fondée sur la restitution et qui prône l'automatisme de l'acte d'écoute. A la lumière de notre réflexion, il apparaît, tout au contraire, que l'interprétation du débat devra s'appuyer sur l'analyse de l'histoire discursive et de la négociation des rôles qui légitiment les prises de positions successives. Cette écoute-là nécessite la mise en place chez l'apprenant d'une capacité à faire le va-et-vient entre les rôles assignés et les rôles revendiqués ainsi qu'entre les rôles et les idées énoncés, tout en tenant compte de la dynamique des interactions et des valeurs sociales implicites. Face à ces enchevêtrements d'implicites constitutifs des stratégies discursives et argumentatives des interlocuteurs, l'apprenant doit pouvoir s'appuyer sur les normes implicites du genre du discours « débat radiophonique » et de ses différentes variantes. Pour ce faire, il faudrait qu'il puisse focaliser son attention sur des marqueurs discursifs qui guideront la reconnaissance de schémas discursifs distincts. Attention et mémoire devront être entrainées afin de suivre les phénomènes en question tout au long du débat pour adapter les jugements aux évolutions qui s'y déroulent.

Sur le plan glottodidactique, la question principale qui se dégage d'une telle analyse est celle du guidage de l'apprenant dans le processus de développement des stratégies requises pour une compréhension fine et critique. Autrement dit: comment, dans quel ordre et dans quelles configurations intégrer tous les niveaux évoqués dans des tâches didactiques? Tout un programme de recherche appliquée, à la croisée de la glottodidactique et de l'analyse du discours, programme ambitieux dont cet article, nous l'espérons, démontre le bien fondé et pour lequel il apporte quelques pistes qui méritent de plus amples investigations. 


\section{BIBLIOGRAPHIE}

Amossy, R. (2010). La présentation de soi. Ethos et identité verbale, Paris : PUF.

Amossy, R.( 2012). L'argumentation dans le discours, Paris : Armand Collin.

Charaudeau, P. (2011). Les médias et l'information, Bruxelles : De Boeck.

Cosnier J. et Kerbrat C. (1987). Décrire la conversation, Lyon : Presses Universitaires de Lyon.

Coste, D., North, B., Sheil, J., Trim, J. (2001). Cadre européen commun de références pour les langues.

Conseil de l'Europe.

Dolz, J., Rey, N., Surian, M. (2004). Le débat : un dialogue avec la pensée de l'autre. Le français aujourd'hui, 146, p. 5-15.

Górecka, J. et Wojciechowska, B. (2013). Rozwijanie rozumienia publicystycznych audycji radiowych na poziomie zaawansowanym : specyfika etapu poprzedzajĄcego słuchanie. Neofilolog, 40/1, p. 59-78.

Górecka, J., Wilczyńska, W. et B. Wojciechowska. (2015). Developing second language oral competence through an integrated discursive approach : The conceptual framework of the project and the pilot study results. In M. Pawlak et E.Waniek-Klimczak (éd.), Theoretical, empirical and pedagogic perspectives on teaching, learning and assessing speaking skills in a second language, Heidelberg Berlin, Springer-Verlag , p. 29-43.

Kerbrat-Orecchioni, C. (1986). L'implicite, Paris : Armand Colin.

Kerbrat-Orecchioni, C. (1990). Les interactions verbales, Paris : Armand Colin.

Perelman, Ch. et Olbrechts-Tyteca, L. (1958). La nouvelle Rhétorique. Traité de l'Argumentation. Paris : Presses Universitaires de France.

Lefeuvre Fl. et Dostie G. (dir.) (à par.) À l'articulation du lexique, de la grammaire et du discours : marqueurs grammaticaux et marqueurs discursifs, Paris : Travaux de linguistique française.

Richard E. \& Drouet G. (sous presse) Confirmer pour mieux détourner : marqueurs d'acceptation et modalités de transition, In : Delahaie, J. et Solis Garcia, I. (éds) Stratégies d'affirmation dans l'interaction, Salerno, Italie : Testi e Linguaggi.

Richard, E., Wojciechowska, B., Le Bot, M.-C., Wilczyńska, W., Górecka, J., Bourvon, M.-F. (2014). Les débats radiophoniques : quelles stratégies d'écoute pour le niveau avancé ? In : C. Martinot et A. Pegaz Paquet (éd.) Innovations didactiques en français langue étrangère, Paris : CRL éditions, p. 38-50.

Wilczyńska, W. et B. Wojciechowska. (2013). Świadomość gatunku dyskusja a rozumienie obcojęzycznych audycji radiowych. Poznańskie Spotkania JĘzykoznawcze. t. XXVI. Poznań : Wydawnictwo PTPN.

Wojciechowska, B., Górecka, J., Richard, E., Le Bot, M.-C. (2014). Les stratégies rédactionnelles face aux défis d'interprétation d'un débat radiodiffusé. Studia Romanica Posnaniensia, XLI/3, p. $117-132$ 
Wojciechowska, B. 2015. « Perception et interprétation des émotions dans le débat radiodiffusé par les étudiants avancés de philologie romane ». Studia Romanica Posnaniensia, XLII/4, Wydawnictwo Naukowe UAM , Poznań, p.155-168.

\section{NOTES}

1. Université Adam Mickiewicz, Département de Philologie romane, Poznań, Pologne.

2. Master Recherche "Nouvelles Recherches en linguistique et en Didactique des langues", Université Rennes 2, Rennes, France, LIDILE EA3874.

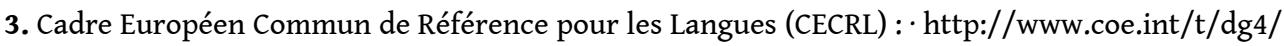
linguistic/source/framework_FR.pdf

4. Rappel des attentes du cadre européen du point de vue de l'implicite (nous soulignons) :

C1 - autonome : l'apprenant, "utilisateur expérimenté », comprend des documents oraux ou écrits longs et exigeants. Il en saisit également l'implicite. Il utilise la langue de façon souple et efficace.

C2 - expert : l'utilisateur comprend tout sans effort ; sa maîtrise de la langue se rapproche de la compétence du locuteur natif.

Il peut comprendre une gamme étendue d'enregistrements audio /vidéo en langue non standard et identifier des détails fins incluant l'implicite des attitudes et des relations des interlocuteurs

Il peut suivre une intervention d'une certaine longueur même si les relations entre les idées sont implicites

5. Voir par ex. Górecka, J. et B, Wojciechowska, 2013, Wojciechowska et al., 2014.

6. Voir par ex. Richard et al., 2014, Wilczyńska et Wojciechowska, 2013, Górecka, Wilczyńska et Wojciechowska, 2015.

7. En outre, les étudiants francophones natifs connaissent ces mêmes défaillances culturelles.

8. On s'aligne ainsi sur les travaux de J. Dolz, N. Rey, M. Surian (2004) qui insistent sur le développement de connaissance de la pratique du débat afin de mieux formuler les objectifs d'enseignement.

9. http://www.franceinter.fr/emission-service-public-nos-politiques-ont-ils-deja-rencontre-lepeuple-0 émission animée par Guillaume Erner avec la participation de trois invités : Raphaëlle Bacqué, grand reporter au quotidien Le Monde, Jacques Julliard, journaliste, écrivain et Michèle Delaunay, députée PS de la Gironde, conseillère municipale de Bordeaux, ancienne ministre déléguée chargée des personnes âgées. La transcription de l'émission est proposée en annexe.

10. Le terme « journaliste " pose difficulté étant donné que ce rôle institutionnel est investi tout au long du débat de façons diverses et débouche sur les rôles communicatifs de journaliste, de modérateur ou d'interviewer. Toutefois pour signaler qu'il s'agit tout le temps du même locuteur nous avons décidé d'utiliser l'étiquette de journaliste.

11. Pour les besoins de ce travail nous adoptons la définition de l'interview proposée par C. Kerbrat-Orecchioni selon laquelle, indépendamment des variantes de l'interview liées à la position et à l'autorité de la personne interviewée et de la personnalité de l'intervieweur, «se caractérise (à la différence de la discussion et du débat) par une dissymétrie des rôles interactionnels, l'intervieweur ayant pour mission d'extirper par ses questions certaines informations de l'interviewé, lequel a pour tâche de les fournir par ses réponses " (KerbratOrecchioni, $1990: 119-120)$.

12. Sur la distinction entre connecteurs et marqueurs on renvoie notamment à Lefeuvre (à par.).

13. R. Bacqué n'utilisera jamais Car, mais Parce que. Preuve supplémentaire de la distinction des postures énonciatives des deux invitées. Une étude suivie, détaillée (argumentative et 
énonciative) de ces marqueurs s'imposerait dans le cadre d'une amélioration de la compréhension orale

14. On en veut pour preuve l'utilisation du verbe "dépeindre » "vous dépeignez finalement un monde fermé ».

15. et on le redit après Ch. Pereleman (1958), M. Doury (2004), R. Amossy (2012).

16. D. Maingueneau (1993: 138).

17. R. Amossy (2012: 94).

18. R. Amossy (2012: 96).

19. Dans cette émission, l'ordre de présentation des invités n'est pas identique à l'ordre que suivra le journaliste pour leur donner la parole. Cela peut aussi être une difficulté pour les apprenants

20. Tous les invités sont des habitués de la parole publique et polémique, en ce sens on peut les considérer comme des experts.

21. Par ailleurs, l'arrivée des femmes dans la politique est ici implicitement présentée comme une source de renouveau de la classe politique.

22. Sur les marqueurs d'accroche à l'initiale d'une prise de parole, on renvoie à Richard $\mathrm{E}$ et Drouet G. (à par.).

23. Michèle Delaunay reprendra la parole pour confirmer et réaffirmer son propre traitement de la problématique et l'installer définitivement dans l'ordre de la réalité, défini comme le professionnel et non le peuple.

Michèle Delaunay : \#2 je veux d'abord dire que dans ce que j'ai écrit c'est exactement ce qui a été dit par Jacques Julliard le besoin d'une diversité professionnelle d'une diversité d'origines et je crois que c'est bien sûr aux partis qui permettent la désignation de l'un de l'autre mais c'est aussi aux électeurs de considérer comme un bonus que le candidat pour lequel ils peuvent voter soit passé par la case réalité par la case vie professionnelle dans tous les milieux \#

\section{RÉSUMÉS}

L'objectif du présent article est de contribuer à la réflexion didactique sur la prise en compte de l'implicite dans le développement des capacités interprétatives relatives au débat radiodiffusé en français langue étrangère. À l'appui de l'analyse d'un débat de l'émission "Service public" deux types d'implicites ont été distingués : 1) les implicites discursifs liés au type de discours (ici le discours médiatique) et au genre du débat polémique, et 2) les implicites socio-discursifs liés aux rôles institutionnels des interlocuteurs, rôles donnés et négociés tout au long du débat radiophoniques.

\section{An interactive negotiation of implicit data in the radio agonistic debate}

This article-aims at contributing to the didactic reflection on the inclusion of implicit data in the development of high interpretative skills of radio debates in French as a foreign language. To support the analysis of a debate from "Service public", a radio segment, two types of the implicit contents were distinguished: 1) implicit elements relating to the type of discourse (media discourse here) and genre agonistic debate and 2) implicit elements relating to the institutional roles of the speakers and the interactive negotiation of these roles in relation to the issue. 
INDEX

Mots-clés : didactique du français langue étrangère, discours médiatique, compétences interprétatives, débat radiodiffusé, identification de l'implicite 